\title{
Gesunde Ernährung: von der Wissenschaft zum Wissen
}

\author{
Bereits zum zweiten Mal initiierte das Forschungsinstitut für \\ Kinderernährung (FKE) gemeinsam mit Kooperationspartnern das \\ Dortmunder Forum für Prävention und Ernährung. Im Mittelpunkt \\ stand das Thema Verhaltensprävention.
}

$\mathrm{P}$ rof. Dr. Mathilde Kersting, stellvertretende Leiterin des FKE, war besonders daran gelegen, eine verbesserte Kommunikation zu erreichen, die über die klassischen Mittel wie Broschüren, Beratung und Vorträge hinausgeht. Dafür seien Kernbotschaften wichtig, die vor allem die besonders betroffenen Kinder aus bildungsfernen Schichten erreichen müssten. Die Tatsache, dass heute bereits $80 \%$ aller Kinder innerhalb von nur drei Tagen zuhause mindestens ein Fertigprodukt konsumieren, erfordert schnelle und nachhaltige Veränderungen in der Ernährungskommunikation, so Kersting.

Sabine Lauxen, Repräsentantin der interministeriellen Arbeitsgruppe „NRW IN FORM“, unterstrich vor allem die Notwendigkeit guter Partner und gut vernetzter Strukturen, um langfristig Ernährungs- und Verbraucherbildung in den Schulen zu verankern. Auch wenn einzelne Programme wie das Schulobstprogramm erfolgreich sind, sei das langfristige Ziel, ein eigenes Ankerfach in der Schule zu etablieren

\section{Einfache Maßnahmen erfolgreich}

„Die beste und billigste Prävention gegen Übergewicht ist nach wie vor das Stillen von Säuglingen", betonte Prof. Dr. Michael J. Lentze, Bonn. Trotz verstärkter Aufklärung habe sich diesbezüglich in den letzten 25 Jahren in Deutschland nur wenig verändert. Damit stehe Deutschland mit seiner "Stillfreudigkeit" noch deutlich zurück hinter Vorzeigeländern aus Skandinavien. In der Ernährungserziehung von Kindern müssten besonders der geringe Gemüseverzehr und der hohe $\mathrm{Zu}$ ckerverzehr (vor allem durch Getränke) berücksichtigt werden. Erfolgreiche Projekte wie das Installieren von Wasserspendern in Schulen (Trinkfit-Studie) konnten zeigen, dass schon mit einfachen Maßnahmen erfolgreiche Prävention möglich ist.

In der Dortmunder Gemeinschaftsverpflegung ist das optimiXKonzept (optimierte Mischkost) des FKE gut angekommen. Dort werden seit 2011 bereits in 25 Schulen Mittagessen nach diesem Konzept eingeführt. OptimiX-Mittagessen werden mit Unterrichtseinheiten zu gesunder Ernährung gekoppelt. Eine besondere Herausforderung in der Grundschule liegt laut Prof. Dr. Dittmar Graf aus Dortmund darin, dass die Fixierung der Geschmackspräferenzen bei Grund-

\section{J1 macht Schule}

Nur etwa ein Drittel der Jugendlichen nutzt die Jugenduntersuchung J1. Ein Projekt klärt jetzt erfolgreich in der Schule über die J1 auf: Wie gewöhne ich mir das Rauchen ab? Wer hilft mir, wenn ich erpresst werde? Dies sind Fragen, die Jugendliche bei der J1 stellen könnten. „Doch wissen sie oft nicht, an wen sie sich wenden können", sagte Simone Linden, Geschäftsführerin von "Mehr Zeit für Kinder". Um die J1 bekannt zu machen, hat Linden mithilfe von Sanofi Pasteur MSD die Initiative „J1 damit du Bescheid weißt" gegründet. Seit Anfang 2011 haben die vier Mitarbeiter Schüler der sechsten und siebten Klassen hessischer Schulen besucht. Dort klären sie nicht nur über die J1 auf, sondern sprechen mit den Heranwachsenden auch über Sexualität, Drogen oder Ernährung. Vorab werden die Ärzte im Umkreis der Schule informiert und erhalten auf Wunsch Plakate für ihre Praxis.

Die J1 sei eine besondere Vorsorgeuntersuchung, weil sie dem Arzt Einblick in

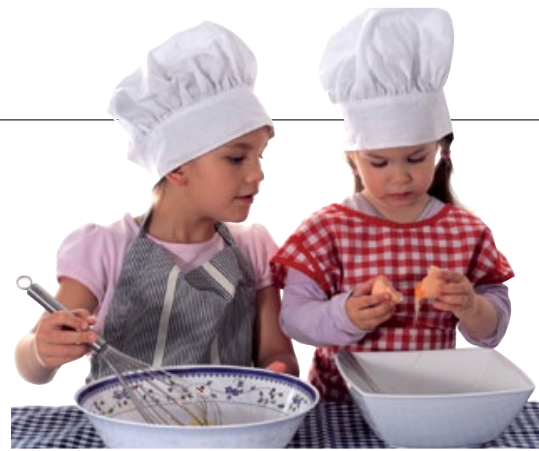

Gemeinsamer Kochunterricht beeinflusst das Essverhalten positiv.

schülern weitestgehend abgeschlossen ist, deren verantwortungsvolles Handeln aber noch nicht vollständig ausgereift ist. Inwiefern sich diese Kopplung von optimiX-Mittagessen und begleitender Unterrichtseinheit positiv auf das Essverhalten der Kinder auswirken wird, werden zukünftige Untersuchungen zeigen.

Klar ist aber jetzt schon, dass man Kinder für das Thema Essen und Ernährung begeistern kann - ob mit dem Wecken ihres Forscherdrangs in einem Labor wie dem Kindertechnologie Zentrum KITZ. do, einer mobilen Küche wie dem Cookie Mobil der Diakonie Dortmund oder gemeinsamen Kochprojekten in der Schule.

Dr. Gunda Backes

2. Dortmunder Forum für Prävention und Ernährung. Dortmund, 29. November 2011
Ressourcen und Resilienz der Jugendlichen gewähre. „Der Satzergänzungstest liefert meistens fantastische Ergebnisse und zeigt häufig schon direkt die Probleme der Betroffenen", sagte Dr. Bernhard Stier vom hessischen Berufsverband der Kinder- und Jugendärzte. Um die Bereitschaft zur J1 zu erhöhen, brauche es aber eine bessere Koordination, sind sich die Podiumsteilnehmer aus Politik, Krankenkassen, Ärzteschaft und der Initiative einig. In Rheinland-Pfalz hat man mit einer staatlichen Einladung zur J1 sehr gute Erfahrungen gemacht. Seit 2008 werden dort Eltern rechtzeitig vor Beginn des Untersuchungszeitraums über die J1 und ihren Nutzen informiert. Seitdem ist die Teilnahmequote um knapp $50 \%$ gestiegen.

Johanna Dielmann-von Berg

Podiumsdiskussion „Vorhang auf für Deutschlands Jugendgesundheit: Bühne frei für die Jugendgesundheitsuntersuchung J1". Wiesbaden, 15. November 2011 